\title{
Importance of IGF-I levels in IVF: potential relevance for growth hormone $(\mathrm{GH})$ supplementation
}

\author{
Norbert Gleicher ${ }^{1,2,3,4}\left(\right.$ D $\cdot$ Sarah K. Darmon ${ }^{1} \cdot$ Emanuela Molinari $^{1} \cdot$ Pasquale Patrizio $^{5} \cdot$ David. H. Barad $^{1,2}$
}

Received: 11 November 2021 / Accepted: 10 December 2021 / Published online: 23 January 2022

(c) The Author(s) 2021

\begin{abstract}
Purpose Growth hormone (GH) supplementation in association with in vitro fertilization (IVF) is worldwide again increasing, even though study outcomes have been discrepant. Since GH acts via insulin-like growth factor-1 (IGF-1), its utilization in IVF would only seem to make sense with low IGF-1. We, therefore, determined whether IGF-I levels affect IVF outcomes. Methods Retrospectively, 302 consecutive first fresh, non-donor IVF cycles were studied, excluding patients on GH supplementation. Patients were divided into 3 subgroups: IGF-1 in lower 25th percentile (group A, $<132 \mathrm{ng} / \mathrm{mL}, n=64$ ); 25th-75th percentile (B, 133-202 ng/mL, $n=164)$, and upper 25 th percentile (C, $>202 \mathrm{ng} / \mathrm{mL}, n=74)$. IGF-1 was tested immunochemiluminometric with normal range at $78-270 \mathrm{ng} / \mathrm{mL}$. Because of the study patients' adverse selection and low pregnancy chances, the main outcome measure for the study was cycle cancellation. Secondary outcomes were oocyte numbers, embryos transferred, pregnancies, and live births.

Results Group A was significantly older than B and C $(P=0.019)$. IGF-1 decreased with increasing age per year by $2.2 \pm 0.65 \mathrm{ng} / \mathrm{mL}(P=0.0007)$. FSH was best in group B and worst in A (trend, $P=0.085)$; AMH was best in B and worst in A (N.S.). Cycle cancellations were lowest in C (11.6\%) and highest in A $(25.0 \% ; P=0.042)$. This significance further improved with age adjustment $(P=0.021)$. Oocytes, embryo numbers, pregnancies, and live birth rates did not differ, though oocyte numbers trended highest in B.
\end{abstract}

Conclusions Here presented results support the hypothesis that IGF-1 levels affect IVF outcomes. GH treatments, therefore, may be effective only with low IGF-1.

Keywords Insulin-like growth factor (IGF-1) · IVF · In vitro fertilization · Cycle cancellations · Infertility

\section{Introduction}

As add-on to ovulation induction for intrauterine inseminations [1] and in vitro fertilization (IVF) stimulation protocols [2], growth hormone $(\mathrm{GH})$ supplementation was

Norbert Gleicher

ngleicher@thechr.com; ngleicher@ rockefeller.edu

1 The Center for Human Reproduction, 21 East 69th Street, New York, NY 10021, USA

2 The Foundation for Reproductive Medicine, New York, NY, USA

3 Stem Cell Biology and Molecular Embryology Laboratory, The Rockefeller University, New York, NY, USA

4 Department of Obstetrics and Gynecology, Medical University of Vienna, Vienna, Austria

5 Department of Obstetrics, Gynecology and Reproductive Sciences, Yale University, New Haven, CT, USA actively utilized for a little over a decade starting in the late 1980s. After a relative hiatus of approximately two decades, GH supplementation has in the last 15 years again become more fashionable [3, 4], even though effectiveness of GH supplementation in improving IVF outcomes has remained controversial $[5,6]$.

$\mathrm{GH}$ is a peptide hormone secreted by anteriorly positioned cells in the pituitary gland (somatotrophs) and plays multiple important roles in the body which go far beyond just support of linear growth, as its name would suggest. Released in pulsatile fashion by GH-releasing hormone with peaks during sleep, it is inhibited by somatostatin, produced in the hypothalamus. Its levels are the highest during puberty and are affected by environmental factors, like sleep patterns, diet, exercise habits, and exposure to stress. The hormone's principal organ target is the liver, where it induces synthesis of insulin-like growth factor (IGF-1) [7]. GH's principal (though not only) activity, therefore, is mediated 
by IGF-1. How GH and IGF-1 affect reproductive tissues has recently been reviewed [8].

Though thus a good number of studies have investigated GH-supplementation in conjunction with IVF, peripheral IGF-1 values in infertile women have been only minimally explored and, indeed, with contradictory findings by the same institution $[9,10]$. Some studies have reported on IGF-1 in follicular fluids and observed correlations to IVF outcomes [11-13].

The GH/IGF-1 signaling pathway (at times also called the somatotropic axis) relates strongly to aging $[12,13]$. In centenarians, functional mutations in the IGF-1-receptor (IGF$1 R$ ), resulting in diminished IGF-1 signaling, are enriched $[14,15]$. In women, low IGF-1 was demonstrated to offer a general survival advantage [16]. As of this point, effects of IGF-1 on ovarian aging are not well defined. Animal data, however, have convincingly demonstrated that GH can stimulate IGF-1 secretion not only from the liver but also from peripheral organs, including ovaries. To complicate matters further, such local IGF-1 secretion can also be stimulated by steroid hormones and/or gonadotropins. Moreover, $\mathrm{GH}$ can also be produced locally in the ovary, in which case the hormone functions in a paracrine, no-pulsatory, and noncircadian mode without involvement of the GH receptor $(G H R)$ [17].

A mouse model, knockout of GHR, however, interestingly did not prevent fertility but reduced litter size [18], thereby delaying exhaustion of the follicle pool [13]. Diminished GH activity in the ovary may, thus, help in maintaining the resting follicle pool (i.e., reduce recruitment), as it naturally declines with advancing female age (i.e., declining functional ovarian reserve). This is also supported by histological examinations, demonstrating a decline in the growing follicle pool. That IGF-1 is, likely, involved in the signaling cascades for these observations is demonstrated by the fact that IGF-1 administration reverses them [19]. Moreover, knockout of the IGF-1 gene in the mouse does results in infertility (and dwarfism), a phenotype that cannot be rescued with gonadotropin stimulation and on histology demonstrates a complete arrest in the development of the growing follicle pool [17]. IGF-1, thus, appears essential for follicle maturation.

Hsu and Hammond in 1987 were the first to demonstrate that GH increased ovarian IGF-1 production in granulosa cells, thereby enhancing FSH action [20]. We today know that $\mathrm{GH}$ and androgens share in this function at small growing follicle stages [21]. With increasing clinical utilization of GH supplementation in IVF, a better understanding of IGF-1 effects on ovaries appears, however, urgently needed. For example, GH supplementation would appear senseless in presence of normal or even high IGF-1 levels, as any hormone supplementation only appears indicated if concentrations in the to be treated microenvironment are insufficient.
It indeed would not surprise if above noted persisting controversy whether GH supplementation improves IVF outcomes may be due to unselected indiscriminate utilization of such supplementation in infertile women. Assuming normal endocrine physiology, GH supplementation should only be effective in women with abnormally low IGF-1 levels.

To elucidate the potential importance of peripheral IGF-1 levels for IVF outcomes, this study, therefore, investigated the importance of untreated initial peripheral IGF-1 levels on IVF cycle outcomes. Results support the hypothesis that peripheral IGF-1 levels relate to IVF cycle outcomes and, therefore, suggest that GH supplementation should only be applied selectively.

\section{Materials and methods}

\section{Study population}

We report on 978 consecutive patients undergoing 815 IVF cycles at our center between 2018 and 2020 who as part of a diagnostic work-up had peripheral IGF-1 level determinations at time of initial consultation. Bloods were routinely obtained approximately 6-8 weeks before IVF cycle start. Patients on GH supplementation and/or in repeat IVF cycles at our center were excluded from this study. Ultimately, 302 fresh first non-donor cycles qualified for the study. Based on IGF-1 levels, these women were then divided into 3 subgroups representing the lower 25th percentile (group A, $<132 \mathrm{ng} / \mathrm{mL}, n=64$ ), the $25 \mathrm{th}-75$ th percentile (group $\mathrm{B}, 132-202 \mathrm{ng} / \mathrm{mL}, n=164)$, and the upper 25 th percentile (group C, $>202 \mathrm{ng} / \mathrm{mL}, n=74$ ), with A considered patients with low, B with normal and $\mathrm{C}$ with high IGF-1 levels.

\section{IGF-1 determinations}

IGF-1 was tested immunochemiluminometric by commercial assay (LabCorp, Burlington, NC), with normal range for all ages defined as $78-270 \mathrm{ng} / \mathrm{mL}$.

\section{Main outcome measures}

Because our center, based on patient age, low ovarian reserve, prior IVF cycles at other centers, and other adverse patient parameters, likely, serves the most adversely selected patient population among IVF centers in the USA (and possibly worldwide), the primary chosen endpoint for the study was cycle cancellations, likely the most sensitive endpoint among patients with high cycle cancellation rates. Secondary study end points were number of oocytes retrieved, embryos transferred, pregnancies, and live births. Because of low expected pregnancy rates, the study was, however, considered underpowered to 
consider them as primary endpoints. Primary and secondary endpoints were also investigated adjusted for patient age at time of presentation. The diagnosis of a clinical pregnancy mandated visualization of pregnancy on vaginal ultrasound examination.

\section{IVF cycle protocol}

As already noted, our center serves a very homogenous, poor-prognosis patient population, characterized by advanced female age, large numbers of prior cycle failures, low functional ovarian reserve, and, therefore, ovarian resistance to stimulation. Patients, consequently, receive individualized ovarian stimulation protocols, which contain the following common denominators: (i) Every woman above age 40 and women below age 40 with LFOR for age and low peripheral androgen levels and/or elevated sex hormone binding globulin (SHBG) receives as previously reported, at least 6-8 weeks of pre-supplementation with dehydroepiandrosterone (DHEA) and CoQ10 prior to IVF cycle start [22]. DHEA supplementation is initiated only after baseline bloods, including IGF-1, are drawn. Cycles are initiated only once androgen levels and SHBG are in normal range. (ii) All cycles are initiated on days 2-3 of menses after ca. 10 days of luteal estrogen supplementation for priming purposes. (iii) Except in younger women with still adequate ovarian reserve, who, per Surrey et al. [23] receive a micro-dose agonist protocol, most patients receive ovarian stimulation without either agonist or antagonist since they receive HIER (highly individualized egg retrieval), with human chorionic gonadotropin (hCG) trigger of 10,000 IU, depending on female age and prior cycle history, at $12-16-\mathrm{mm}$ lead follicle size [24, 25]. Because of the early egg retrieval, agonists/antagonists to prevent spontaneous ovulation are not required in such patients. (iv) All patients receive gonadotropin stimulation of 450-600 IU per day, usually at 3:1 ratio of FSH to human menopausal gonadotropin (hMG) products (manufacturers vary, depending on patient preference and/ or insurance coverage). If patients have a history of very poor prior response to such stimulation, they in parallel also receive Clomiphene citrate $100 \mathrm{mg}$ for 5 days, starting on day 2 of menses. (v) Considering the importance of every embryo in this patient population, the embryology laboratory performs, as also previously reported, rescue in vitro maturation of every immature oocyte [26].

(vi) Cryopreservation of embryos is as much as possible avoided and patients preferably undergo fresh transfers. (vi) Cycles utilizing autologous oocytes are always transferred at cleavage stage and transfers are performed under ultrasound control. (vii) Pregnancy test is obtained 12 days following embryo transfer.

\section{IRB approval}

Since this study only involved data extraction from our center's anonymized electronic medical research data base, it only required expedited IRB approvals. Every included patient provided written permission by consent to utilize their medical records for research purposes, as long as their anonymity was maintained, and the medical record remained confidential.

\section{Statistical analyses}

Continuous variables were presented with mean \pm standard deviation and compared between IGF-1 groups by an ANOVA test. Categorical variables were compared between IGF-1 groups with Fisher's exact test. Age was compared to continuous IGF-1 levels by linear regression. Logistic regression and negative binomial regression models were used to adjusted for patients' age. A $P$-value $<0.05$ was considered statistically significant. Analyses were performed by the center's medical statistician (S.K.D.) using SAS version 9.4 (SAS Institute, Cary, NC).

\section{Results}

The distribution of IGF-1 levels in the whole study population was Gaussian (Fig. 1a). Patients in the lowest IGF-1 quartile (group A) were significantly older $(43.0 \pm 4.8$ years) than those in mid-range (group B, $41.3 \pm 4.9$ years) and highest quartile group $\mathrm{C}(40.7 \pm 5.6$ years; $P=0.019)$. This is of importance because, as one would expect, IGF-1 levels were age dependent: A linear regression revealed that IGF-1 levels decreased with increasing age $2.2 \pm 0.65 \mathrm{ng} / \mathrm{mL}$ per year $(P=0.0007$; Fig. $1 b)$.

Table 1 demonstrates further details: Though not statistically different, trends reflecting ovarian reserve parameters were the best in group B: FSH was $17.3 \pm 17.8$ vs. in group A, $24.8 \pm 35.3$ and in group C, $18.1 \pm 20.6 \mathrm{mIU} / \mathrm{mL}$; $P=0.085 ;$ AMH was $1.4 \pm 3.3$ vs. in group $\mathrm{A}, 0.7 \pm 1.2$ and in group $\mathrm{C}, 1.0 \pm 1.6 ; P=0.200$ ).

Cycle cancellations were statistically the lowest in $\mathrm{C}$ (11.6\%), the highest in A (25.0\%), and in mid-range in B (13.5\%; $P=0.042)$. Oocyte numbers, transferred embryos, pregnancy, and live birth rates did not differ significantly, though oocyte numbers trended the highest in group B $(5.2 \pm 5.4$ years $)$ vs. $3.6 \pm 5.4$ in group $\mathrm{A}$ and $4.5 \pm 5.0$ in group C. Adjusting statistical assessments for age, the difference in cancelled cycles became even more significant $(P=0.021)$, while all other outcome, likely because of too small patient numbers, remained non-significant. 
(a)

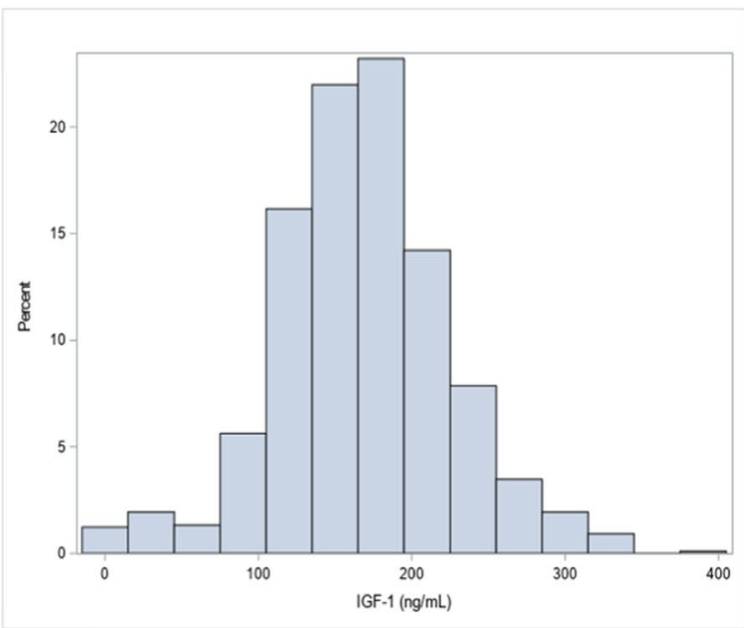

(b)

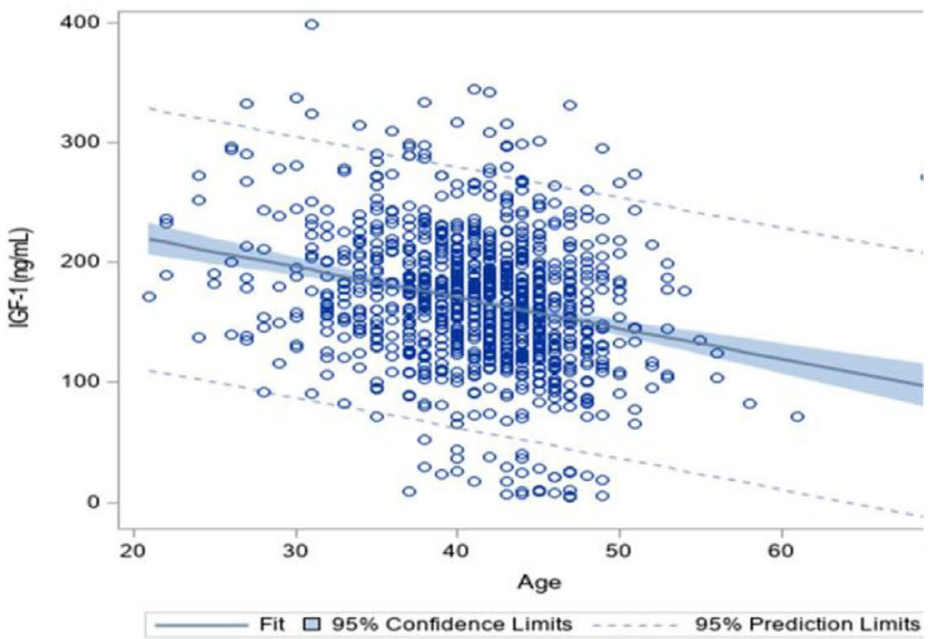

Fig. 1 a Demonstrates the Gaussian distribution of IGF-levels in the study population. b Reflects the linear progression, demonstrating the age dependency of IGF-1 $(P=0.0007)$

Table 1 Patient differences between groups $\mathrm{A}, \mathrm{B}$, and $\mathrm{C}$

\begin{tabular}{llllll}
\hline & Group A & Group B & Group C & $P$-value & $\begin{array}{l}P \text {-value } \\
\text { adjusted for } \\
\text { age }\end{array}$ \\
\hline$N$ & 64 & 164 & 74 & & \\
Age (years) & $43.0 \pm 4.8$ & $41.3 \pm 4.9$ & $40.7 \pm 5.6$ & 0.0191 & \\
AMH (ng/mL) & $0.7 \pm 1.2$ & $1.4 \pm 3.3$ & $1.0 \pm 1.6$ & 0.1995 & \\
FSH (mIU mL) & $24.8 \pm 35.3$ & $17.3 \pm 17.8$ & $18.1 \pm 20.6$ & 0.0845 & \\
Cycles & & & & & \\
$\quad$ Cancelled cycles & $16(25.0 \%)$ & $10(13.5 \%)$ & $9(11.6 \%)$ & 0.0421 & 0.0212 \\
$\quad$ Oocytes retrieved & $3.6 \pm 5.4$ & $5.2 \pm 5.4$ & $4.5 \pm 5.0$ & 0.1274 & 0.1870 \\
$\quad$ Embryos transferred & $1.1 \pm 1.5$ & $1.5 \pm 1.4$ & $1.4 \pm 1.5$ & 0.1668 & 0.1184 \\
$\quad$ Pregnancies (\%) & $2(3.1 \%)$ & $9(5.5 \%)$ & $4(5.4 \%)$ & 0.8258 & 0.8518 \\
$\quad$ Live births (\%) & $0(0 \%)$ & $7(4.3 \%)$ & $2(2.7 \%)$ & 0.2796 & 0.7765 \\
\hline
\end{tabular}

\section{Discussion}

It is important to initiate the discussion of here presented results by pointing out one more time the highly unfavorable selection of here presented patient population (Table 1). Not only were patients of advanced age, from a mean of 40.7 years in group C, 41.3 years in group B to a mean of 43.0 years in group A $(P=0.019)$, but they also demonstrate highly unfavorable functional ovarian reserve parameters, with $\mathrm{FSH}$ in this case demonstrating the best abnormal median in group B at $17.3 \mathrm{mIU} / \mathrm{mL}$, group C with FSH $18.1 \mathrm{mIU} / \mathrm{mL}$ holding the middle, and group A with $24.8 \mathrm{mIU} / \mathrm{mL}$ being the worst, though differences did not reach significance $(P-0.085)$. They, however, correlated with abnormally low AMH levels, with group B again demonstrating the best mean level of $1.4 \mathrm{ng} / \mathrm{mL}$, followed by group $\mathrm{C}$ at $1.0 \mathrm{ng} / \mathrm{mL}$ and group A again demonstrating the by far poorest mean value at $0.7 \mathrm{ng} / \mathrm{mL}$, though these differences were statistically also not significant.

Despite quite a large number of first IVF cycles (at our center) in this study $(n=302)$, because of the unfavorable prognosis of here investigated patient, pregnancy and live birth rates were as expected relatively low (Table 1). This can be assumed to be a reason why oocyte numbers retrieved, numbers of transferrable embryos and pregnancy, and live birth rates did not reach statistical significance between study groups. Cycle cancellation rates, clearly the most sensitive outcome parameter in poor prognosis patients, however, did demonstrated statistically significant differences between study groups based on IGF-1 level and 
these differences even strengthened with age adjustment. Further studies, involving even larger patient numbers as well as better prognosis patients, will, however, be helpful in reaching more definite answers as to why, even in most unfavorable IVF patients, cycle cancellations do statistically relate to IGF-1 levels.

Since cycle cancellation rates in this study clearly inversely correlated with IGF-1 levels, this study for the first time offers a potential selection tool for women in infertility treatments who may benefit from GH supplementation in association with IVF. All evidence points toward women in group B (normal IGF-1 levels) demonstrating best outcomes. This finding, alone, supports the study's initial hypothesis that GH supplementation may improve IVF outcomes only in patients with low IGF-1 levels (group A). These findings potentially also explain the very conflicting results in the literature regarding GH utilization in association with IVF, as unselected utilization will, of course, dilute effectiveness of GH treatment: Just as aspirin will relive headache only in patients with headache and will be ineffective in a general population without a preponderance for headache, so will GH only be effective in women with low IGF-1 levels, through which $\mathrm{GH}$ exerts its physiological effects on ovaries.

Our results to a degree contradict studies from a single laboratory, claiming in two studies poorer IVF cycle outcomes with increasing IGF-1 levels [10, 27]. The same group in an earlier study, however, as we do here, reported highest cycle cancellations with lowest IGF-1 and lowest cancellations with highest IGF-1 [9]. Their most recent study involved so-called poor-responders but ages were clearly younger and FSH and AMH levels more favorable than in our patient population [27]. In addition, these authors defined high IGF-1 levels as anything over $72.0 \mathrm{ng} / \mathrm{mL}$, while in our study, even the lowest 25 th percentile was going as high as $132 \mathrm{ng} / \mathrm{L}$. These two studies, therefore, are not comparable and an insightful accompanying editorial noted that drawing conclusions from this group's recent study for several additional reasons was difficult [28]. For these reasons and because of physiological logic, here observed statistical correlation between low IGF-1 and increased IVF cycle cancellation risk, therefore, is credible.

At absolute minimum, GH supplementation, thus, appears indicated in women with low peripheral IGF-1 levels, in this study defined as $<132 \mathrm{ng} / \mathrm{mL}$ (lower 25 th percentile). As noted earlier in the "Introduction" section of this manuscript, positive effects of GH supplementation should not surprise in absence of $\mathrm{GH}$ and especially of adequate IGF-1 levels [13, 18-20]. Though such supplementation has remained controversial $[5,6]$, our improving understanding of GH/IGF-1 effects on granulosa cells and the resulting synergism with FSH effects on follicle growth support such supplementation but only if it occurs in women with low IGF-1 levels. We, therefore, propose that future studies of
GH supplementation in IVF cycles should be preceded by IGF-1 evaluations and only women with abnormally low levels should be considered for such supplementations.

Here presented findings are, however, also interesting for their apparent contradictions: On the one hand, there appears strong evidence for a beneficial effect of IGF-1 on IVF cycle completion; yet, while the positive effect on cycle completion appears linear with increasing IGF-values, functional ovarian reserve, as represented by FSH and AMH levels, on the other hand, appears best at mid-levels of IGF-1 (group B). Cycle cancellations as well as FOR are clearly the worst in group A, also the oldest patients in this study and, therefore, are not a surprise. Reaffirming the likelihood of a causal association with IGF-1, age, however, does not appear to explain cycle cancellations since significance was maintained (and actually improved) after age adjustments $(P=0.021)$.

Cycle cancellations automatically denote IVF cycle failure. Though a statistical association does not establish causation, here demonstrated statistical association between IGF-1 levels and IVF outcomes strongly supports a causal relationship since this association even strengthened after age adjustments. How, specifically, IGF-1 lowers cycle cancellation risks, remains to be established. Cycle completion mandates at least one oocyte and one transferrable embryo. One, therefore, may conclude from here presented findings that better IGF-1 levels support the likelihood that at least one embryo becomes available for transfer. IGF-1, may achieve this by, as previously noted, enhancing recruitment [29] and acting synergistically with androgens and FSH in follicle maturation during small growing follicle stages [21]. Improvements in egg and embryo numbers after GH supplementation have, indeed, also been reported in studies that have failed to demonstrate improvements in pregnancy and live birth rates $[5,6]$ and, therefore, based on existing literature appear as of this point factual.

Whether there are other ways by which the GH-IGF-1 axis may beneficially influence IVF outcomes remains as of this point unsettled. An aged mouse model, recently reported by Chinese investigators, offers complementary information to here presented data: In that study, the authors confirmed that GH increased the number of antral follicles and of retrieved oocytes most at a medium dosage, second-best at high dosage and least at low dosage. This effect was achieved in those animals without obvious changes in AMH levels. Because improvements also correlated with increasing ATP levels, frequency of homogenous mitochondrial distribution, and improved mitochondrial membrane potential (though not with mtDNA copy numbers), the authors suggested that GH improved mitochondrial function in oocytes [30]. GH, in addition, also appears effective in improving in vitro maturation of human oocytes [31, 32]. Finally, recent studies also strongly hint at effects of $\mathrm{GH}$ on endometrial receptivity 
[32] which, thus, potentially appears to offer an independent contribution to improved IVF outcomes from ovarian $\mathrm{HGH} /$ IGF-1-effects.

After female age, egg and embryo numbers in a given IVF cycle represent the second most-important predictor of pregnancy and live birth chances in IVF [33]. They in that same study also related in a rather peculiar way to $\mathrm{AMH}$ levels that may also have some relevance to here reported results: As pregnancy and live birth rates increased with larger egg and embryo yields, they did so also in parallel to increasing AMH levels. That increase, however, persisted only up to a certain AMH level, at which point, with further increasing $\mathrm{AMH}$, not only did pregnancy rates start declining but miscarriage rates skyrocketed. Beyond certain AMH thresholdlevels, its initially positive effects on IVF outcomes, thus, turned radically negative. IGF-1 may demonstrate a similar effect-reversal with increasing concentrations in the peripheral circulation, as Irani et al. in frozen-thawed IVF cycles recently reported higher miscarriage rates associated with higher peripheral IGF-1 levels [34]. This observation further supports above noted suspicion that this group of investigators dealt with a very different patient population with quite different IGF-1 cut offs in comparison to this study,.

Here presented IGF-1 data, suggesting best FOR at midrange for IGF-1 (in our study at roughly 132-202 ng/mL), are supported by above noted mouse study [30], suggesting similar IGF-1 dynamics, with a "best" level at mid-range. Endocrinology is defined by "best" endocrine ranges for practically all hormones. Another good example in control of ovarian function is androgen levels, with too low and too high, producing subpar IVF outcomes [21].

\section{Limitations, summary, and conclusions}

The highly unfavorable patient populations our center serves obviously limits the applicability of here reached conclusions (Table 1). Considering the advanced age and low functional ovarian reserve of all three here reported patient groups, IVF outcomes were characterized by relatively small oocyte yield, embryo numbers, and few pregnancies. Consequently, it is not surprising that, despite a reasonably large patient population, no significant differences were observed in secondary IVF cycle outcome parameters. Though difficult to assess considering the various outcome parameters, our statistician concluded that study groups would at least have to double in size to also demonstrate differences in other clinical IVF cycle outcome parameters than cycle cancellations. Though advanced ages of the study population must be carefully considered before generalizing here observed findings to younger age groups, that age adjustment actually improved the significance of here reported finding, in a way validates them.
This study clearly supports further exploration of $\mathrm{GH}$ supplementation especially in women with low IGF-1 levels, usually mostly older patients. While ovaries in younger women may reveal different hormonal dynamics, we, therefore, would not be surprised if younger women with low IGF-1 levels would also be positively affected by supplementation with $\mathrm{GH}$.

Two additional issues deserve mention: As IGF-effects on ovaries are most profound at small growing follicle stages, follicles exposed to adequate IGF-1 levels still require at least 6-8 weeks to reach gonadotropin-dependence that renders them available to gonadotropin stimulation in IVF cycles. GH supplementation must, therefore, be started at least 6-8 weeks before IVF cycle start. A large majority of studies in the medical literature supplemented patients with $\mathrm{GH}$, however, only during stimulation or, at best, starting about 2 weeks before stimulation start. Such supplementation, like androgen supplementation which supports follicle growth with identical timing [21], will not result in desired effects on granulosa cells of growing follicles (and, therefore, oocytes), though they may, at right concentrations, exert beneficial endometrial effects [35, 36]. Second, the literature also varies greatly in daily dosages of $\mathrm{GH}$ that were administered. Here, too, a consensus must be reached if study outcomes are to be compared.

As a final message, this manuscript also suggests that determination of IGF-values, generally not considered a routine test in infertility practice, may be indicated in women with low functional ovarian reserve.

Author contribution Study concept: N.G., P.P., D.H.B.; data accumulation: S.D., E.M., D.H.B.; statistics: S.D., D.H.B.; first manuscript draft: N.G.; manuscript revisions: all authors; financial and overall study responsibility: N.G.; all authors approved of the final version of the manuscript.

Funding This work was supported by intramural funds from the Center for Human Reproduction and partial grant support from the Foundation for Reproductive Medicine, a not-for-profit research foundation, both located in New York, NY.

Data availability Data and materials are upon reasonable request available from The CHR's data depository by contacting Ms. Jolanta Tapper, COO at jtapper@thechr.com/.

\section{Declarations}

Ethics approval and consent to participate This study was approved by the IRB of The CHR. All patients in this study signed an informed consent that allowed use of their medical record for research purposes if their identity was protected, and the material remained confidential. Since this study involved only use of deidentified electronic medical records, these conditions were met.

Consent for publication Above noted approval also includes a consent for publication. 
Competing interests N.G. and D.H.B. are listed as co-owners of several already awarded and still pending US patents, some claiming benefits from androgen supplementation in women with low functional ovarian reserve, a topic peripherally addressed in this manuscript. Others relate to diagnostic and potential therapeutic benefits of AMH, also marginally addressed in this manuscript. N.G. is a shareholder in Fertility Nutraceuticals, LLC, which produces a DHEA product, and is owner of The CHR., where much of the research reported in this manuscript was performed. N.G. and D.H.B. also receive patent royalties from Fertility Nutraceuticals, LLC. N.G., P.P., and D.H.B. also received research support, travel funding, and lecture fees from various Pharma and medical device companies, none, however, over the last 3 years and none in any way related to this manuscript. Other authors have no conflicts to report.

Open Access This article is licensed under a Creative Commons Attribution 4.0 International License, which permits use, sharing, adaptation, distribution and reproduction in any medium or format, as long as you give appropriate credit to the original author(s) and the source, provide a link to the Creative Commons licence, and indicate if changes were made. The images or other third party material in this article are included in the article's Creative Commons licence, unless indicated otherwise in a credit line to the material. If material is not included in the article's Creative Commons licence and your intended use is not permitted by statutory regulation or exceeds the permitted use, you will need to obtain permission directly from the copyright holder. To view a copy of this licence, visit http://creativecommons.org/licenses/by/4.0/.

\section{References}

1. Homburg R, Eshel A, Abdalla HI, Jacobs HS. Growth hormone facilitates ovulation induction by gonadotrophins. Clin Endocrinol (Oxf). 1988;29(1):113-7.

2. Owen EJ, Ostergaard H, Shoham Z, Jacobs HS. Cotreatment with growth hormone, after pituitary suppression, for ovarian stimulation in in vitro fertilization: a randomized, double-blind, placebocontrolled trial. Fertil Steril. 1991;56(6):1105-10.

3. Li X-L, Wang L, Fang LV, Huang X-M, Wang L-P, Pan Y, Zhang $\mathrm{X}-\mathrm{M}$. The influence of different growth hormone addition protocols to poor ovarian responders on clinical outcomes in controlled ovary stimulation cycles. A systematic review and meta-analysis. System Rev Meta-Analyses. Medicine. 2017;96:12.

4. Yovich JL, Regan SLP, Zaid S, Keane KN. The concept of growth hormone deficiency affecting clinical prognosis in IVF. Front Endocrinol. 2019;19(650):1-9.

5. Xu Y-M, Hao G-M, Gao B-L. Application of growth hormone in in vitro fertilization. Front Endocrinol. 2019;10(502):1-11.

6. Zhu J, Wang Y, Chen L, Liu P, Li R, Qiao J. Growth hormone supplementation may not improve live birth rates in poor responders. Front Endocrinol. 2020;11:1-9.

7. Hart RJ. Use of growth hormone in the IVF treatment of women with poor ovarian reserve. Front Endocrinol (Lausanne). 2019;10:500.

8. Ipsa E, Cruzat VF, Kagize JN, Yovich JL, Keane KN. Growth hormone and insulin-like growth factor action in reproductive tissues. Front Endocrinol (Lausanne). 2019;10:1-14.

9. Ramer I, Kanninen TK, Sisti G, Witkin SS, Spandorfer SD. Association of in vitro fertilization outcomes with circulating insulinlike growth factor components prior to cycle initiation. Am J Obstet Gynecol. 2015;213(3):356.e1-6.

10. Nasioudis D, Minis E, Irani M, Kreines F, Witkin S, Spandorefer S. Insulin-like growth favtor-1 and soluble FMS-like tyrosine kinase-1 prospectively predict cancelled IVF cycles. J Assist Reprod Genet. 2019;36(12):2485-91.

11. Genc G, Yilmaz N, Uygur D. The effect of intrafollicular IGF 1 and IGFBP 3 on IVF outcome in patients using different gonadotropins: a prospective study. J Assist Reprod Genet. 2011;28:405-10.

12. Barzilai N, Huffman DM, Muzumdar RH, Bartke A. A critical role of metabolic pathways in aging. Diabetes. 2012;61:1315-22.

13. Bartke A, Quainoo N. Impact of growth hormone -related mutations on mammalian aging. Front Genet. 2018;9:586. https://doi. org/10.3389/fgene.2018.00586.

14. Suh Y, Atzmon G, Cho MO, Hwang D, Liu B, Leahy DJ, Barzilai N, Cohen P. Functional significant insulin-like growth factor I receptor mutations in centenarians. Proc Nat Acad Sci USA. 2008;105:3438-42.

15. Tazearslan C, Huang J, Barzilai N, Suh Y. impaired IGF1R signaling in cells expressing longevity-associated human IGF1R alleles. Aging Cell. 2011;10:551-4.

16. Milamn S, Atzmon G, Huffman DM, Wan J, Crandall JP, Cohen P, Barzilai N. Low insulin-like-growth factor 1 level predicts survival in humans with exceptional longevity. Aging Cell. 2014;13:769-71.

17. Dosouto C, Calaf J, Polo A, Haahr T, Humaidan P. Growth hormone and reproduction: Lessons learned from animal models and clinical trials. Front Endocrinol. 2019;10:1-7.

18. List EO, Sackman-Sala L, Berryman DE, Funk K, Kelder B, Gosney ES, Okada S, Ding J, Cruz-Topete D, Kpchick JJ. Endocrine parameters and phenotypes of te growth hormone receptor gene disrupted (GHR-/-) mouse. Endocr Rev. 2011;32(3):356-86.

19. Slot KA, Kastelijn J, Bachelot A, Kelly PA, Binart N, Teerds KJ. Reduced recruitment and survival of primordial and growing follicles in GH receptor-deficient mice. Reprod Camb Engl. 2006;131:525-32.

20. Hsu CH, Hammond JM. Concomitant effects of growth hormone on secretion of insulin -like growth factor I and progesterone by cultured porcine granulosa cells. Endocrinology. 1987;121:1343-8.

21. Prizant H, Gleicher N, Sen A. Androgen actions in the ovary: Balance is key. J Endocrinol. 2014;222(3):R141-151.

22. Gleicher N, Barad DH. Dehydroepiandrosterone (DHEA) supplementation in diminished ovarian reserve (DOR). Reprod Biol Endocrinol. 2011;9:67.

23. Surrey ES, Bower J, Hill DM, Ramsey J, Surrey MW. Clinical and endocrine effects of a microdose GnRH agonist flare regimen administered to poor responders who are undergoing in vitro fertilization. Fertil Steril. 1998;69(3):419-24.

24. Wu Y-G, Barad DH, Kushnir VA, Lazzaroni E, Wang Q, Albertini DF, Gleicher N. Aging-related premature luteinization of granulosa cells is avoided by early oocyte retrieval. J Endocrinol. 2015;226(3):167-80.

25. Wu Y-G, Barad DH, Kushnir VA, Wang Q, Zhang L, Darmon SK, Albertini DH, Gleicher N. With low ovarian reserve, highly individualized egg retrieval (HIER) improves IVF results by avoiding premature luteinization. J Ov Res. 2018;11(1):23. https://doi.org/10.1186/s13048-018-0398-8.

26. Lee H-J, Barad DH, Kushnir VA, Shohat-Tal A, LazzaroniTealdi E, Wu Y-G, Gleicher N. Rescue in vitro maturation (IVM) of immarure oocytes in stimulated cycles in women with low functional ovarian reserve (LFOR). Endocrine. 2016;52(1):165-71.

27. Man L, Levkovich J, Canon C, Rosenwaks Z. Cycle day2 insulinlike growth factor-1 serum levels as a prognostic tool to predict controlled ovarian hyperstimulation outcomes in poor responders. Fertil Steril. 2020;113(6):1205-14. 
28. Forman EJ. In search of a new biomarker to predict poor ovarian response. Fertil Steril. 2020;113(6):111.

29. Bartke A, Chandrashekar V, Dominici F, Turyn D, Kinney B, Steger R, Kopchick JJ. Insulin-like growth factor 1 (IGF-1) and aging: controversies and new insights. Biogerontology. 2003;4:1-8.

30. Hou H-Y, Wang X, Yu Q, Li H-Y, Li S-J, Tang R-Y, Guo Z-X, Chen Y-Q, Hu C-X, Yang Z-J, Zhang W-k, Qin Y. Evidence that growth hormone can improve mitochondriak fucntion in oocytes from aged mice. Reproduction. 2019;157:345-58.

31. Hassan HA, Azab H, Abdel Rahman A. Effects of growth hormone on in vitro maturation of germinal vesicle of human oocytes retrieved from small antral follicles. J Assist Reprod Genet. 2001;18:417-20.

32. Altmäe S, Aghajanova L. Growth hormone and endometrial receptivity. Front Endocrinol. 2019;10:1-11. https://doi.org/10.3389/ fendo.2019.00653.

33. Gleicher N, Kushnir V, Sen A, Darmon SK, Weghofer A, Wu Y-G, Wang Q, Zhang L, Albertini DF, Barad DH. Definition by FSH, AMH and embryo numbers of good-, intermediateand poor prognosis patients suggests previously unknown IVF outcome-determining factor associated with AMH. J Transl Med. 2016;14:172.

34. Irani M, Nasioudis D, Witkin SS, Gunnala V, Spandorefer S. High serum IGF-1 levels are associated with pregnancy loss following frozen-thawed euploid embryo transfer cycles. J Reprod Immunol. 2018;127:7-10.

35. Li Y, Liu H, Yu Q, Liu H, Huang T, Zhao S, Ma J, Zhao H. Growth hormone promotes in vitro maturation of human oocytes. Front Endocrinol. 2019;10:1-8. https://doi.org/10.3389/fendo. 2019.00485.

36. Liu F-T, Wu Z, Yan J, Norman RJ, Li R. The potential role of growth hormone on the endometrium in assisted reproductive technology. Front Endocrinol. 2020;11:1-8. https://doi.org/10. 3389/fendo.2020.00049.

Publisher's note Springer Nature remains neutral with regard to jurisdictional claims in published maps and institutional affiliations. 